\title{
"ANÁLISIS COMPARATIVO DE LA ESCALA DE JUEGO PRE-ESCOLAR DE KNOX REVISADA (RKPPS) Y TEST DE DESARROLLO PSICOMOTOR 2-5 AÑOS (TEPSI), DESDE LO CULTURALMENTE SEGURO, EN VALDIVIA, DURANTE EL AÑO 2013"
}

"COMPARATIVE ANALYSIS BETWEEN THE REVISED KNOX PRESCHOOL PLAY SCALE (RKPPS) AND THE TEST DE DESARROLLO PSICOMOTOR 2-5 AÑOS (TEPSI) FROM A CULTURALLY SAFE PERSPECTIVE, IN VALDIVIA, DURING THE YEAR 2013"

\section{Valentina Cardemil Figueroa', Natalia Quilodrán Sanhueza², Catalina Soto Gutiérrez² .}

\begin{abstract}
Resumen:
El presente estudio fue llevado a cabo con el objetivo de analizar y comparar los elementos de la seguridad cultural en la aplicación de los instrumentos de evaluación Test de desarrollo psicomotor 2-5 años (TEPSI) y Escala de Juego pre-escolar de Knox (RKPPS) aplicados por Terapeutas Ocupacionales en población pre-escolar valdiviana. Dicha investigación fue realizada a través de un enfoque cualitativo, con el abordaje metodológico de Teoría Fundamentada, realizando entrevistas semi-estructuradas a cuatro Terapeutas Ocupacionales que aplican ambos instrumentos. Como resultado se obtuvo que ninguno de los dos instrumentos se acercan al espectro de lo culturalmente seguro, sin embargo, es la RKPPS la que incorpora más elementos de dicha conceptualización. Finalmente se concluye que los profesionales de la disciplina se ven desafiados a permanecer en la dicotomía de, por una parte, utilizar instrumentos cuantitativos para validarse ante otros profesionales y tener un lenguaje común, y por otro lado, instrumentos cualitativos para realizar intervenciones integrales con usuarios. Esa elección se ve influenciada por una serie de variables, dadas por las distintas demandas en su desempeño laboral, impactando en su forma de evaluar.
\end{abstract}

\section{Palabras Clave:}

Instrumentos de Evaluación, Test de Desarrollo Psicomotor 2-5 años, Escala de Juego de Knox, culturalmente seguro, pre-escolares.

\begin{abstract}
:
The following study was developed with the intention of analysing and comparing the elements of cultural safety in the application of these two assessment tools, the "Revised Knox Preschool Play Scale" (RKPPS) and the "Test de Desarrollo Psicomotor 2-5 Años"(TEPSI) on preschool Valdivian population by Occupational Therapists. This research was conducted through a qualitative approach, using Grounded Theory as methodological approach, and semi-structured interviews to four Occupational Therapists that regularly use both assessment tools were done. As a result, neither the RKPPS nor the TEPSI, are close to the culturally safe spectrum. However, the RKPPS is the one that embodies more elements from this conceptualization. Finally, it has been concluded that professional OTs are being challenged to remain in this dichotomy: On the one hand, using quantitative tools to validate themselves in front of other professionals and in order to have a common language; on the other hand, using qualitative tools to do comprehensive interventions with clients. This choice is influenced by a series of variables, due to different demands in their job performance, impacting on their way of evaluating.
\end{abstract}

\section{Keywords:}

Assesment tools, Test de Desarrollo Psicomotor 2-5 años (TEPSI), Revised Knox Preschool Play Scale (RKPPS), cultural safety, Preschool children. 


\section{INTRODUCCIÓN}

Desde los comienzos de la Terapia Ocupacional se ha reconocido la importancia de intervenir no sólo en las ocupaciones que realizan las personas, sino también en el medio en el que está inserto el individuo. Teniendo en cuenta que el contexto socio-cultural de un sujeto impacta en las ocupaciones que elige y en la razón de dicha elección, ello entregará patrones de cómo la persona piensa, siente y actúa de acuerdo a la cosmovisión que tiene desde su contexto socio-cultural.

Es así como, dentro del proceso de atención en salud, se ha reconocido la importancia de la pertinencia cultural, siendo la cultura un fenómeno que trasciende lo exclusivamente étnico, pues implica valorar la diversidad biológica, cultural y social del ser humano como un factor importante en todo proceso de salud y enfermedad (Alarcón, Vidal y Neira, 2003). Es por ello que surge en la década de 1980 el concepto de Seguridad Cultural, impulsado por enfermeras de origen Maorí en Nueva Zelanda (Smye, Josewski, \& Kendall, 2010). Ellas propusieron que la atención en salud es culturalmente segura cuando "se reconoce, respeta y nutre la identidad cultural única de las personas, y cuidadosamente se conocen sus necesidades, expectativas y derechos" (p. 8).

El concepto de Seguridad Cultural considera variados principios básicos, uno de ellos indica que lo culturalmente seguro se mueve más allá del concepto de la sensibilidad cultural, lo que implica analizar los desequilibrios de poder, la discriminación institucional, la colonización y las relaciones con los colonizadores que existen en los servicios de salud. A lo anterior se suma la necesidad de que los profesionales de la salud deben interactuar con los clientes, de tal manera que aquellos que reciben atención en salud, deben definirla (Iwama, 2007).

Dentro del mismo orden de cosas, dentro de la Terapia Ocupacional, también han surgido acercamientos a la postura de lo culturalmente seguro. Uno de estos puntos de vista es el de Michael Iwama (2006), quien define cultura como "una compleja interacción de significados compartidos que representan y forman las vidas individuales y colectivas de las personas" (p. 19).

Por otro lado, en relación a los procesos de evaluación en Salud, en el contexto chileno, específicamente en la población pre-escolar (niños de 2 años a 5 años 11 meses), se aplican diversos instrumentos de evaluación que cotejan distintas destrezas de los niños y niñas. Estos permiten determinar el nivel de desarrollo en el que se encuentran los infantes de acuerdo a su edad cronológica. Producto de la relevancia que toma el desarrollo de los niños/as en edad pre-escolar en Chile, el Ministerio de Educación en conjunto con el Ministerio de Salud, promueven la evaluación de variados aspectos del desarrollo normativo de los menores, a través de instrumentos validados en nuestro país, como lo es el Test de desarrollo psicomotor 2-5 años, de aquí en adelante TEPSI, creado en Chile en el año 1985 y estandarizado con población de la Región Metropolitana y Quinta Región (Haeussler y Marchant, 2003). Esta herramienta tiene como propósito "conocer el desarrollo psíquico en tres áreas básicas: Motricidad, Coordinación y Lenguaje y realizar un "screening" o tamizaje detectando en forma gruesa riesgos o retrasos en el desarrollo" (p. 3).

Por otra parte, una de las premisas fundamentales de la Terapia Ocupacional es que "el juego es una ocupación significativa y fundamental" (Pacciulio, Pfeifer \& Santos, 2010). Basada en dicha idea, en el año 1968, la Terapeuta Ocupacional Susan Knox crea en Estados Unidos un instrumento que permite evaluar diversas habilidades por medio de la observación del juego libre en diferentes contextos. La llamada "Escala de juego pre-escolar de Knox, Revisada" (RKPPS) de aquí en adelante Escala de Knox, diseñada para evaluar a niños de entre 0 a 6 años, valora habilidades motrices finas y gruesas, intereses, lenguaje y participación en el medio físico y social (Knox, 1997). De esta manera, permite que el terapeuta identifique las áreas específicas de juego en las que el menor presenta dificultades y necesita de intervención.

Las pautas anteriormente mencionadas, tienen en común algunos aspectos tales como: ambas son utilizadas en población pre-escolar; permiten realizar seguimiento al usuario/a; se requiere de la presencia del niño o niña para aplicar dichos instrumentos y ambas ofrecen información cuantitativa y cualitativa de las habilidades que poseen los infantes, lo cual nos puede ayudar a completar el proceso de evaluación en contextos clínicos. Sin embargo resulta relevante destacar que "los instrumentos utilizados en la medición del desarrollo no son realmente transculturales. Esto, porque la mayor parte de ellos han sido diseñados pensando en las habilidades que la sociedad occidental promueve" (Bedregal, 2008, p. 32). Adicionalmente, Iwama (2003) argumenta que gran parte del discurso de los profesionales de la terapia ocupacional se encuentra asentado en la cosmovisión occidental, la que se caracteriza por 
tener una estructura positivista, racional y empírica (p. 3), lo cual privilegiaría una visión individualista del ser humano, quien se encontraría necesariamente separado de su medio ambiente. Es por todo lo anterior, que creemos necesario realizar un análisis del grado de seguridad cultural de los mencionados instrumentos.

Vale la pena destacar que producto de toda la información expuesta y sumado al análisis realizado, es que de aquí en adelante se entenderá por práctica culturalmente segura como el respeto y reconocimiento de la persona con sus características e intereses, dentro de su contexto físico, institucional y sociocultural, invitándolo a tomar decisiones, siendo el sujeto partícipe de su proceso salud-enfermedad, donde además el Terapeuta Ocupacional debe ser capaz de reconocer su propia cultura y actitudes, y su potencial para ejercer poder sobre los usuarios. De esta manera, se comprenderá por la aplicación de instrumentos de forma culturalmente segura en niños en edad pre-escolar, el uso de estas herramientas considerando el contexto y flexibilizando la metodología, sin perjuicio de los objetivos que busca la evaluación. Ello, apelando al conocimiento y criterio del profesional que utilice el instrumento, de modo de priorizar las necesidades del niño, rescatando sus habilidades y capacidades en pos de su desempeño ocupacional.

Finalmente, el objetivo de la siguiente investigación es analizar y comparar los elementos de la seguridad cultural presentes en la aplicación de los instrumentos de evaluación TEPSI y Escala de Knox aplicados por Terapeutas Ocupacionales en población pre-escolar valdiviana, durante el año 2013.

\section{MetodoloGía}

La presente investigación fue elaborada a través de un enfoque cualitativo; este enfoque estima la importancia de la realidad, tal y como es vivida por las personas, sus ideas, sentimientos y motivaciones; intenta identificar, analizar, interpretar y comprender la naturaleza profunda de las realidades (Rivas, 2006). Esto permitió reconocer elementos desde lo culturalmente seguro que se puedan evidenciar en la aplicación de los instrumentos de evaluación en población pre-escolar por Terapeutas Ocupacionales. Esto es posible debido al carácter naturalista e interpretativo de este enfoque.
De la misma forma, tanto el estudio como los resultados se abordaron desde la Teoría Fundamentada o Grounded Theory. Dicha metodología utiliza un procedimiento sistemático cualitativo para generar una teoría que explique en un nivel conceptual una acción, una interacción o un área específica (Hernández, Fernández, y Baptista, 2010). Se optó por este diseño metodológico debido a la escasa evidencia existente en relación al grado de Seguridad Cultural en la aplicación de instrumentos de evaluación en pre-escolares en Chile.

Por otro lado, la técnica de recolección de información que resultó pertinente para acercarse al objetivo de estudio de esta investigación, es la entrevista semiestructurada, la cual es entendida como un diálogo "en donde el entrevistado mantiene la conversación enfocada sobre un tema particular, y le proporciona al informante el espacio y la libertad suficiente para definir el contenido de la discusión" (Tarrés, 2001, p. 76).

La investigación realizada arrojó resultados que se agruparon en una serie de categorías en un primer nivel de análisis, obtenidas a través de la metodología de codificación abierta, utilizando el método de análisis línea por línea, el cual fue producto de la transcripción y comparación de las entrevistas realizadas, obteniendo como conclusiones las categorías presentadas posteriormente en la discusión del estudio.

Las Terapeutas Ocupacionales seleccionadas para formar parte del presente estudio, debían cumplir con los siguientes criterios: trabajar o haber trabajado con población pre-escolar, aplicar o haber aplicado el TEPSI y la Escala de Knox, y que se encontraran ejerciendo sus labores en la ciudad de Valdivia. Se entrevistó a cuatro profesionales de la disciplina, siendo todas las consultadas mujeres, con edades que varían desde los 28 a los 42 años, y con años de experticia que van desde los 5 a los 19. Dos de las entrevistadas se desempeñan en contextos educacionales de carácter público (Jardín infantil y escuela especial), mientras que las dos restantes ejercen sus labores en contextos privados, tanto en consulta particular como clínica privada. Por otra parte, todas las consultadas egresaron de universidades pertenecientes al consejo de rectores, siendo dos de ellas egresadas de la Universidad de Chile y las restantes de la Universidad Austral de Chile. Tres de las entrevistadas ejercen su profesión en equipos interdisciplinarios, mientras la restante se desempeña de manera individual. Cabe destacar que todas llevan al menos dos años desempeñando labores terapéuticas dentro del mismo contexto institucional. 


\section{Resultados}

\section{(A) "Antigüedad del instrumento".}

Este aspecto no se encuentra presente en el discurso de las entrevistadas al referirse a la Escala de Juego Preescolar de Knox, pero sí al hablar del TEPSI, ya que este instrumento se encontraría descontextualizado en relación a los elementos socio-temporales de la época y las características de la población actual. Esto se relaciona con dos aspectos:

1. La antigüedad del proceso de validación y estandarización.

2. La antigüedad del material.

Desde este punto de vista, las consultadas destacan las características particulares que tiene la población infanto-juvenil actualmente, la cual, según una de las mismas, "ha cambiado, los niños son distintos, y también lo son los medios de estimulación".

\section{(B) "La importancia del contexto"}

En relación a este elemento, se evidenciaron resultados discrepantes con respecto a ambos instrumentos. Por una parte, en relación al TEPSI, las entrevistadas mencionaron que esta evaluación no considera aspectos del contexto de aplicación de la herramienta, y además, posiciona al usuario en un ambiente artificial, dado en las instrucciones de uso de la pauta, debido a que en éstas se indica, apartar al niño de su ambiente habitual, lo cual generaría mantener al usuario en una situación estresante.

En relación a la Escala de Knox, se menciona dentro de los resultados, como un aspecto positivo de la evaluación, la consideración dentro de sus ítems y objetos de evaluación, los materiales y el ambiente en que el niño se desempeña en la actividad de juego. Sin embargo, otra entrevistada menciona que el requerimiento de un contexto determinado, donde el niño pueda jugar libremente y tener acceso a materiales de juego, puede resultar ser un impedimento para la aplicación del instrumento en algunos contextos institucionales, al no contar con dichas condiciones.

\section{(C) "Variaciones en la metodología"}

Otro de los resultados obtenidos se relaciona con variaciones en la metodología de aplicación de los instrumentos, particularmente del TEPSI, ya que a pesar de que dicha evaluación es una pauta estructurada, muchas personas la aplican de maneras inadecuadas y su uso se ve fuertemente influenciado por la visión profesional que tenga el examinador.

De esta manera, las entrevistadas indicaron la necesidad de flexibilizar la aplicación del instrumento, de modo de hacerlo más acorde a la situación del usuario. Como dice una de ellas:

"En mi opinión, el instrumento debiera, basado en una entrevista o en el conocimiento que tú tengas del contexto del niño, tener la flexibilidad respecto de qué materiales utilizar (...). Tendría que ser flexible, porque si ahora establecieras 'el material del TEPSI actualizado' es lo mismo, porque en el fondo generas otra instancia rígida de evaluación".

Además, una de las consultadas indica que existe escasa capacitación en el uso del TEPSI, lo cual podría interferir en el resultado de la evaluación y su posterior interpretación.

Con respecto a la Escala Knox, se identificó que, al no encontrarse traducida al español y estandarizada en nuestro país, puede hacerse un uso más libre de su aplicación, de modo de acomodar los elementos del instrumento a la situación del niño. Sin embargo, una de las consultadas menciona lo siguiente:

"Yo creo que habría que diseñar un Knox con los materiales que efectivamente los niños suelen utilizar en Valdivia o en el lugar donde tú estés".

\section{(D) "Entendimiento del concepto de lo culturalmente seguro"}

Durante las entrevistas, se consulta a las profesionales respecto al concepto de lo culturalmente seguro, lo cual arrojó dos ideas principales:

1. Las terapeutas mencionan que incorporan no solo a la persona y a su contexto en el proceso de intervención, sino que, además, toman 
en cuenta la experiencia adquirida y el razonamiento profesional que resulta de ello.

2. Por otra parte, se menciona dentro de la narrativa de las entrevistadas, que para hablar de un instrumento culturalmente seguro, este debe romper con la separación sujeto-objeto.

En palabras de una entrevistada:

"Estos instrumentos están constituidos bajo un paradigma positivista en que hay un estándar, hay una separación sujeto-objeto. Entonces, vas con tipo de lente a observar a otro distinto a ti, por lo tanto, tú eres un sujeto que observa desde afuera y eso no existe, porque te constituyes como otro al momento de evaluar".

Con respecto al instrumento TEPSI, las entrevistadas mencionan que este no es una evaluación culturalmente segura, puesto que no considera las características de los niños, sus intereses o su contexto. En cambio, en relación a la Escala de Knox, el hecho de que evalúa las habilidades de los pre-escolares en un contexto de juego libre, genera resultados que no serían posibles de apreciar por medio de una prueba estructurada, lo cual permitiría incorporar parte de lo culturalmente seguro en el proceso de evaluación. Sin embargo, es importante considerar que, según una de las entrevistadas, la posibilidad de observar el juego libre varía en función de las oportunidades que entrega el ambiente en relación al uso de espacio y materiales, lo cual igualmente alejaría al instrumento de la conceptualización de lo culturalmente seguro.

\section{(E) "Fundamentos del uso de los instrumentos"}

Las entrevistadas señalan que el principal motivo por el cual utilizan el TEPSI es porque éste se encuentra validado y estandarizado. También señalan que es una pauta clara y conocida, lo que permite compartir los resultados con otros profesionales. Además mencionan que les permite sintetizar, sistematizar y orientarse respecto a las habilidades observadas en un niño o niña de acuerdo a su etapa evolutiva.

Con respecto a la Escala de Knox, las opiniones concuerdan con que es beneficioso evaluar al niño/a con un instrumento de la Terapia Ocupacional, donde el usuario es evaluado en instancias de juego libre.

\section{(F) "Modificaciones a los instrumentos"}

En primer lugar, en relación al instrumento TEPSI, las entrevistadas mencionan que la principal modificación que realizarían a la evaluación sería una actualización del mismo, además de incorporarle una perspectiva centrada en la ocupación. De la misma manera, en relación a la Escala de Knox, las entrevistadas mencionan la necesidad de modificar algunos rangos de edad, incorporando las características de los niños/ as valdivianos. Finalmente, se destaca la importancia de flexibilizar ambos instrumentos en relación al uso de materiales y contexto, lo cual ayudaría a rescatar una mayor cantidad de información referente a las habilidades que se desean observar.

\section{(G) "Relaciones de poder y origen cultural del propio conocimiento"}

El concepto de lo culturalmente seguro incorpora las relaciones de poder y la cultura donde se enmarca el proceso de intervención, el cual determinaría la calidad del vínculo y el proceso terapéutico. Como resultado del proceso de entrevistas, es posible aseverar que las consultadas toman en cuenta el poder que ejercen sobre los usuarios al momento de realizar una evaluación. En palabras de una entrevistada:

"No puedo entender cómo es posible hacer Terapia Ocupacional sin saber lo que es el otro. Entonces la seguridad cultural también apela a ver en el otro lo que esa persona es, y lo que necesita y quiere. Ahora lo que uno ve en los niños es aun más acentuado, porque se supone que ellos, culturalmente, no toman decisiones, no tienen poder para determinar lo que es lo más idóneo para ellos, entonces ahí es que yo creo que va el cambio, en facilitar el acceso a la elección de los niños, a partir de las fortalezas que ellos tienen para desarroIlar el juego".

\section{DISCUSIÓN}

\section{1) Respeto y reconocimiento de la persona con sus características e intereses: Formación de vínculo terapéutico y empoderamiento del usuario.}

Producto del análisis realizado, es posible rescatar que, previo a realizar cualquier tipo de evaluación, es 
necesario establecer un vínculo terapéutico con el/la usuario/a, lo cual implica que el evaluador debe reconocer al otro como un ser único, con sus características e intereses, y con la capacidad de empoderarse del proceso de evaluación, pudiendo negarse a ejecutarla, o prefiriendo involucrarse en otra actividad. Ello estaría incluido en la literatura, al hacer mención al concepto de lo culturalmente seguro, ya que para "reconocer, respetar y nutrir la identidad cultural única de las personas, y conocer sus necesidades, expectativas y derechos" (Smye, Josewski, \& Kendall, 2010, p. 8), se debe tener un conocimiento íntegro del usuario y su situación, donde además el terapeuta debe reconocer su propia cultura y actitudes.

Lo anterior se destaca en las entrevistas cuando las consultadas se refieren al TEPSI, pues al tratarse, según las mismas, de una prueba estructurada de 'screening', puede resultar altamente estresante, y sus resultados pueden ser distintos si el niño conoce o no al examinador, lo cual habla de las distintas variables que debe manejar el evaluador antes de aplicar el instrumento y del escaso reconocimiento que tiene el Test de las características y expectativas del niño/a. Todo lo anterior discrepa con la perspectiva planteada por Anastassi y Urbina (1998), quienes mencionan que "si los resultados que obtienen distintas personas en una prueba han de ser comparables, las condiciones del examen tienen que ser las mismas para todos, producto de que en dicha situación, la única variable independiente, es a menudo, el individuo examinado" (p. 6), lo cual se encuentra en completa oposición a lo sugerido por los autores de la línea de lo culturalmente seguro.

Por otro lado, en cuanto a la Escala de Knox, producto de que fue "desarrollada para proporcionar una descripción de la conducta de juego libre" (Knox, 1997, p. 784), el cual se caracteriza por "ser intrínseco, espontáneo, divertido, absorbente, vitalizante, desafiante, noliteral y un fin en sí mismo" (p. 35), es posible observar que la escala entrega la posibilidad de respetar los intereses de juego (tipo de juego), los materiales (juguetes preferidos) y el contexto. Ello implicaría que este instrumento, en esencia, respeta y reconoce mayormente al niño con sus características e intereses, acercándose al espectro de lo culturalmente seguro.

\section{2) Consideración del contexto sociocultural, físico e institucional}

En relación al contexto, las entrevistadas mencionan que según las instrucciones del TEPSI, dicha evaluación se debe realizar de manera individual (en un espacio donde solo se encuentre el examinador y el niño) y en un contexto libre de distracciones, siendo necesario apartarlo del grupo donde el/la usuario/a se desenvuelve habitualmente, para poder obtener resultados válidos y confiables (Haeussler y Marchant, 2003, p. 56). Es por ello que este instrumento dejaría fuera aspectos contextuales valiosos y enriquecedores desde la valoración de lo culturalmente seguro, que pudieran estar impactando en el desempeño del menor, ya que según lo que menciona Iwama (2007), "aquello que es concebido como verdadero no se presenta como singular y universal, sino, más bien, es visto como múltiple y cambiante de acuerdo a los contextos particulares de cada situación" (p. 3).

Por otra parte, en relación a la Escala de Knox, tal como se mencionó anteriormente, la aplicación de la evaluación en el contexto de juego libre del niño, sería un aporte significativo del instrumento, pues dentro de su estructura considera el ambiente del juego y sus materiales, tomando en cuenta que Reilly define el juego como un sistema multidimensional de adaptación al medio ambiente donde la conducta exploratoria de curiosidad subyace al comportamiento del juego (Knox, 1997). Sin embargo, es importante destacar que, como requisito para la aplicación de la Escala, el contexto de aplicación del instrumento debe dar al niño la oportunidad de desempeñarse en juego libre, con una variedad de juguetes de interés a su disposición. Lo anterior se podría transformar en una limitación, dependiendo de los contextos en los cuales se aplique la evaluación.

\section{3) Criterio del profesional que utiliza el instrumento}

En cuanto a esta categoría, es posible referir que según mencionan las entrevistadas, el criterio del profesional es un factor que repercute profundamente en la evaluación e intervención. Además, dentro del proceso de evaluación, intervención y seguimiento se apela continuamente a dicho criterio profesional para lograr respetar los derechos y la opinión del usuario/a. En relación a la aplicación del TEPSI, el aporte de los datos cualitativos depende del profesional, debido a que siempre pueden encontrarse, pero incorporarlos o no 
dentro del proceso de evaluación depende directamente del examinador.

Por otra parte, es posible extraer de los testimonios reportados, que el instrumento tiene ciertas características que entregan poder al evaluador, promoviendo que se produzca una relación vertical y por consiguiente, una separación sujeto-objeto, lo cual iría en desmedro de una visión desde lo culturalmente seguro ya que dicha conceptualización implica "analizar los desequilibrios de poder, la discriminación institucional, la colonización y las relaciones con los colonizadores que existen en los servicios de salud" (Iwama, 2007).

En cuanto a la aplicación de la Escala de Knox, las entrevistadas mencionan que recae en el criterio del observador el poder adaptar el material, orientar el juego libre y generar un ambiente provechoso para la evaluación, lo cual de igual manera impacta no sólo en dicho proceso, sino que durante todo el proceso de intervención.

Lo anteriormente mencionado coincidiría con lo que mencionan los autores Jankovich, Mullen, Rinear, Tanta \& Deitz (2008), quienes entregan una serie de recomendaciones para modificar el instrumento de acuerdo al contexto y al criterio del profesional, apelando a la traducción cultural de la evaluación.

\section{4) Flexibilización de la metodología sin alterar los objetivos de los instrumentos}

En este ámbito, destaca dentro de las entrevistas la necesidad inherente de realizar una adecuada capacitación en relación a la forma de aplicación de dichos instrumentos, debido a la posibilidad de flexibilizar la metodología en relación a las diversas áreas de evaluación de la pauta, esto sin perjuicio de los objetivos. Sin embargo, cabe destacar que el TEPSI establece que la administración del "test debe efectuarse en forma idéntica a la señalada en el manual de administración. No deben agregarse motivaciones adicionales, comentarios u otros que hagan que la situación de medición varíe de examinador a examinador" (Haeussler y Marchant, 2003 , p. 56). Además, las instrucciones de administración refieren que el test debe administrarse completo a cada niño y en el orden estipulado (p. 57). Todo lo anterior sugeriría la incompatibilidad de este instrumento con el concepto de lo culturalmente seguro.

Por otra parte, en la Escala de Knox es posible adaptar la duración de su aplicación, ya que si bien, de acuerdo a las instrucciones, el niño debe ser observado durante dos sesiones de 30 minutos en dos ambientes diferentes, uno interior y otro exterior, sin embargo, se ha encontrado que es posible utilizar dos sesiones de 15 minutos (Jankovich, Mullen, Rinear, Tanta \& Deitz, 2008, p. 223). Lo anteriormente señalado implicaría que la forma de administración de la Escala de Knox podría estar sujeta a cambios según las necesidades del niño/a. Por otra parte, las entrevistadas refieren que al no encontrarse estandarizada en el país, permitiría que el instrumento pueda aplicarse con mayor flexibilidad, tomando en cuenta tanto las características de la pauta como los objetivos que la misma persigue, haciendo que ésta se acerque mayormente a la realidad en la que participa el niño.

\section{5) Reconocimiento del Terapeuta Ocupacional de su propia cultura y del poder que puede ejercer sobre los usuarios}

Como ya se mencionó anteriormente, lo culturalmente seguro alude al reconocimiento por parte del profesional de su cultura y actitudes, así como también del potencial poder que puede ejercer sobre los usuarios (Iwama, 2007). Es así que a lo largo de las entrevistas, queda en claro que las profesionales entrevistadas tienen posicionamientos y opiniones claras respecto a los instrumentos por los cuales se les consulta. A su vez, se observa que tienen alguna noción respecto a lo que significa la seguridad cultural y su respectiva importancia en el trabajo del Terapeuta Ocupacional. Sin embargo, sólo una de ellas reconoce abiertamente las características culturales de la ciencia con la que se relaciona cotidianamente. Esto puede deberse a lo que menciona Gerlach (2012) en su trabajo, ya que "la claridad conceptual de la seguridad cultural puede estar en riesgo por el aumento de su aplicación, en las diferentes disciplinas de la salud a diversos grupos a nivel internacional" (p. 3). A su vez, la misma autora justifica lo anterior producto de que la manera en que se traducen los fundamentos críticos de la seguridad cultural a los entornos de práctica sigue siendo ambigua.

Posterior al análisis realizado en la presente investigación, en primer lugar es posible concluir que, si bien el posicionamiento de las entrevistadas respecto a los instrumentos es discrepante en diversos temas, éste también varía en función del contexto laboral en que se desempeñan las Terapeutas Ocupacionales. Este ambiente incluiría diversos ámbitos, tales como el equipo de trabajo, el espacio físico y las características globa- 
les de las instituciones, los cuales generarían distintas demandas al desempeño laboral, impactando en su forma de evaluar.

En cuanto al TEPSI, es posible mencionar que este instrumento no cumple con los criterios para el concepto de lo culturalmente seguro, debido a que no incorpora el contexto en la evaluación, es rígido en su aplicación, y omite las características e intereses de los usuarios, ubicándolos en una posición de desventaja, producto de que es el evaluador quien tiene el poder durante la ejecución del instrumento. Sin embargo, cabe destacar que el objetivo de dicha evaluación es realizar un 'screening' del desarrollo de los niños, incorporando solo aspectos cuantitativos en la realización del instrumento. No obstante, al ser estandarizado abre la puerta al diálogo con otras disciplinas tanto del área de la salud, como de educación, pudiendo validar las experiencias profesionales en función de dicha evaluación y complementar las visiones apuntando a una intervención integral. Además, cabe destacar que en nuestro país es necesario cuantificar los resultados de evaluación a través de instrumentos estandarizados, para poder optar y justificar la necesidad de recursos públicos para la atención de niños en edad pre-escolar.

Por otra parte, la Escala de Knox logra acercarse al concepto de seguridad cultural, debido a que toma en cuenta el contexto en el cual se desenvuelve el niño o niña durante el juego libre y da al examinador la oportunidad de flexibilizar la metodología de aplicación. Sin embargo, a pesar de lo anterior, es necesario tomar en cuenta que, para la correcta aplicación del instrumento, se requiere de la posibilidad de propiciar un ambiente que permita al niño jugar libremente, con los materiales de interés y la participación de terceros que se involucren en la evaluación. Lo anterior dependería principalmente de dos factores: el razonamiento y criterio del profesional, y el contexto en el cual se encuentre ejerciendo su labor.

No obstante, es necesario dejar en claro que independiente del instrumento utilizado, las entrevistadas consideran que es positivo que al posicionarnos como evaluadores, seamos capaces de rescatar una mayor cantidad de información del tipo cualitativo que permita fortalecer y enriquecer la evaluación de los pre-escolares, tomando en cuenta su contexto y acercándose a la seguridad cultural.

Al mismo tiempo, es posible concluir que los Terapeutas Ocupacionales se encuentran constantemente enfrentados a una dicotomía en su quehacer cotidiano: Por una parte el uso de herramientas cuantitativas, de modo de poder validarse profesionalmente frente a otras disciplinas y poder tener un lenguaje común; y la incorporación de elementos cualitativos, para realizar intervenciones integrales. Cabe destacar que la incorporación de elementos de una u otra herramienta, se ve altamente influenciada por el contexto laboral en que se desempeñan los Terapeutas Ocupacionales, debido a lo que permite o restringe dicho contexto institucional. Además, la decisión tomada por los/as Terapeutas también se ve impactada por el criterio profesional que poseen los mismos, lo cual varía en función de las características y el proceso de tratamiento de los niño/as, incorporando el contexto en el cual se desenvuelve el usuario y la intervención.

Cabe señalar que la visión de este estudio no apunta a construir nuevos instrumentos de evaluación que cumplan con el concepto de culturalmente seguro. Esto requeriría de una gran cantidad de pasos, considerando la variedad de culturas y contextos presentes en Chile, y tomando en cuenta las diversas variables que ésta debería considerar para cumplir con la conceptualización. La importancia de ser capaces de adquirir la perspectiva de lo culturalmente seguro dentro de los procesos de evaluación e intervención radica en que, en el futuro, los profesionales de la disciplina deben ser capaces de generar instancias de conversación, discusión y debate, con la finalidad de flexibilizar el uso de los instrumentos y rescatar la cultura de los usuarios, de modo de generar evaluaciones que se acerquen al espectro de lo culturalmente seguro, considerando el contexto en el cual se involucran, sus características y las necesidades e intereses de los niños y niñas. Junto con esto, es necesario trabajar no solo con la mirada de los Terapeutas Ocupacionales, sino que también, es importante adentrarse en el mundo de otras disciplinas de manera colaborativa, para lograr posicionarnos como un equipo de salud desde una perspectiva amplia y segura.

Por otra parte, se desprende del presente estudio que en relación a la seguridad cultural, esta investigación no podría tener validez en otras poblaciones, debido a que no necesariamente se relaciona nuestra cultura con otras. Además hay que tener en cuenta que, en una primera instancia, se había propuesto que la muestra cuente con un número mínimo de diez entrevistadas, la cual debió disminuirse. De la misma manera, las entrevistadas finalmente fueron Terapeutas Ocupacionales mujeres, y de universidades del Consejo de rectores, lo cual también generó una perspectiva limitada de información respecto a lo consultado. 
Finalmente uno de los problemas posibles de abordar en el futuro, sería la realización de una investigación similar, que incorpore Terapeutas Ocupacionales de ambos sexos, y que se desempeñen laboralmente en contextos rurales, lo cual puede aportar con otra mirada a la perspectiva propuesta en la presente investigación. Además, podría resultar interesante incorporar dentro de un estudio de características similares, la visión de otros profesionales, como educadores/as, enfermeras, y otros trabajadores del área de la salud, particularmente su opinión respecto al TEPSI, instrumento ampliamente utilizado en sus contextos laborales.

\section{RefERENCIAS BIBLIOGRÁfICAS}

Alarcón, A.; Vidal, A. y Neira, J. (2003). Salud Intercultural: elementos para la construcción de sus bases conceptuales. Revista Médica de Chile, 131, 1061-1065.

Anastassi, A. y Urbina, S. (1998). Naturaleza y Uso de las pruebas psicológicas. En A. Anastassi y S. Urbina. Tests Psicológicos (pp. 2-31). México: Prentice Hall.

Bedregal, P. (2008). Instrumentos de medición del desarrollo en Chile. Revista Chilena de pediatría, 79(1), 32-36.

Gerlach, A. (2012). A critical reflection on the concept of cultural safety. Canadian Journal of Occupational Therapy, 79, 151-158.

Haeussler, I. y Marchant, T. (2003). TEPSI: Test de desarrollo psicomotor 2-5 años. Santiago, Chile: Ediciones U. Católica de Chile.
Hernández, R.; Fernández, C. y Baptista, P. (2010). Metodología de la Investigación ( $5^{\mathrm{a}}$ ed.). México: McGraw-Hill.

Iwama, M. (2003). Toward Culturally Relevant Epistemologies in Occupational Therapy. American Journal of Occupational Therapy, 57(5), 582-588.

Iwama, M. (2006). The Kawa Model, Culturally Relevant Occupational Therapy. Churchill Livingstone Elsevier.

Iwama, M. (2007). Embracing diversity: Explaining the cultural dimensions of our occupational therapeutic selves. New Zealand Journal of Occupational Therapy, 54(2), 16-23.

Jankovich, M.; Mullen, J.; Rinear, E.; Tanta, K. \& Deitz, J. (2008). Revised Knox Preschool Play Scale: Interrater agreement and construct validity. American Journal of Occupational Therapy, 62, 221-227.

Knox, S. (1997). Development and current use of the knox preschool play scale. En L. Parham \& S. Fazio, Play in occupational therapy for children. (pp. 35-50). United States, St. Louis: Mosby.

Pacciulio, A.; Pfeifer, L. \& Santos, J. (2010). Preliminary reliability and repeatability of the Brazilian version of the revised Knox preschool play scale. Occupational Therapy International, 17, 74-80.

Rivas, M. (2006). Reseña de la investigación cualitativa etnográfica en educación. Manual teórico-práctico. Revista venezolana de educación, 10(35), 757-758.

Smye, V.; Josewski, V. \& Kendall, E. (2010). Cultural Safety: an Overview. Extraído el 3 de octubre de 2012 desde http://www. mooddisorderscanada.ca/documents/Publications/CULTURAL\%20SAFETY\%20AN\%20OVERVIEW\%20(draft\%20 mar\%202010).pdf.

Tarrés, M. (2001). Observar, escuchar, y comprender: sobre la tradición cualitativa en la investigación social. D.F., México: Universidad autónoma Metropolitana. 